\title{
Selection of Imprinted Nanoparticles by Affinity Chromatography
}

\author{
António R. Guerreiro*, Iva Chianella, Elena Piletska, Michael J. Whitcombe, Sergey A. \\ Piletsky \\ Cranfield Health, Cranfield University, MK43 0AL, UK \\ * E-mail address: a.guerreiro@ cranfield.ac.uk
}

\begin{abstract}
Soluble molecularly imprinted nanoparticles were synthesised via iniferter initiated polymerisation and separated by size via gel permeation chromatography. Subsequent fractionation of these particles by affinity chromatography allowed the separation of high affinity fractions from the mixture of nanoparticles. Fractions selected this way possess affinity similar to that of natural antibodies $\left(K_{d} 6.6 \times 10^{-8}\right) \mathrm{M}$ and were also able to discriminate between related functional analogues of the template.
\end{abstract}

Keywords: molecularly imprinted polymer; nanoparticle; affinity chromatography

\section{Introduction}

The development of synthetic materials which mimic biological systems by design or by function has become a hot topic in modern science (Mosbach et al., 1996, Zimmerman et al., 2003, Wulff et al., 2006). Among the typical representatives of synthetic receptors are molecularly imprinted polymers (MIPs) which possess enormous potential for the development of a new generation of stable biomimetic materials (Holthoff et al., 2007). However, the main drawback of this technology is the formation of a diverse population of binding sites, ranging from low to high affinity, mainly due to thermodynamic reasons (Wulff et al., 1977). 
Nanoparticles are practically always formed during the early stages of MIP synthesis, which is performed using a relatively high percentage of cross-linkers (60 - 90\%). Typically, following initiation and primary chain growth, the polymerization reaction proceeds through several stages (Figure 1). Small branched polymers are formed by monomer addition from solution (a). Highly branched molecules (b) are then formed during the early stages by intra-molecular reaction of growing polymer chains and by chain extension due to addition of new monomers or polymer from solution. As reaction progresses, larger chains are formed and more reactive groups are trapped inside the molecule, favouring intramolecular cross-linking. At this stage highly cross-linked macromolecular clusters with stable network structure (c) are formed (Li et al., 2004). If the reaction is allowed to proceed, then molecular clusters react with each other forming large globules (d). Continuing association process together with chain growth will lead to the formation of insoluble polymer (e). Termination of the reaction at stage (c) leads to the formation of soluble nanoparticles. This has been achieved in the past by dilution of monomer mixture prior to gelation, using free radical initiators with thermal initiation (Wulff et al., 2006). At this stage however reaction progresses very quickly and precise control over the size of particles is difficult to achieve when using UV initiation, which was used here to prepare the nanoparticles. Better control over the reaction kinetics can be attained using slower iniferter-mediated polymerization. However the fundamental advantage of using this form of living polymerization, as compared with free radical polymerization, lies in the possibility to subsequently re-initiate polymerization by exposure to UV radiation, where the polymer nanoparticles will act as a macroinitiator molecule. The terminating groups dissociate to form polymeric radicals (Peppas et al., 2004) which can attack vinyl groups resulting in extra polymeric growth or covalent immobilization of the particle on a surface containing these groups. This simple approach can be used to easily immobilize the nanoparticles on sensor surfaces or to induce sequential functionalization (e.g. signaling functionality) of the particles, increasing the scope for practical applications of this type of materials. The nanoparticle approach can offer a unique solution to the problems frequently encountered in MIP technology 
associated with the presence of low affinity binding sites. Each MIP microparticle (polymer bead or obtained by grinding a monolith) contains many binding sites, with a range of different affinities. If however we start to shrink particle size, then in theory we should be able to select the ones with "good" binding sites. For this type of material we can use affinity chromatography with immobilised template to capture particles containing a greater number of relatively high affinity sites. Here we demonstrate the synthesis of MIP nanoparticles combined with affinity chromatography. The product of this process has affinity comparable to that of natural antibodies, whilst allowing for the removal of particles with low affinity.

\section{Materials and Methods}

Due to its length, this section is presented as Supplementary Material.

\section{Results and Discussion}

\subsection{Preparation of naoparticles}

For proof of concept we have chosen a model based on polymers imprinted with 2,4-diamino-6methyl-1,3,5-triazine, common name acetoguanamine (see Supplementary Material). The affinity adsorbent for separation of high affinity nanoparticles was prepared using a polymerisable analogue of the template, 2,4-diamino-6-(methacryloyloxy)ethyl-1,3,5-triazine (DMET), see structures on Figure 3. Triazines were successfully imprinted before using similar compositions (Muldoon et al., 1997, Sergeyeva et al., 1999). Optimal results were obtained using a mixture of two cross-linkers: ethylene glycol dimethacrylate (EGDMA) and trimethylolpropane trimethacrylate (TRIM), which provided the right balance of flexibility/rigidity which is crucially important for the subsequent molecular recognition. Control polymers were prepared in the absence of template. The nanoparticles produced were characterized by size exclusion chromatography (SEC) as described previously (Sun et al., 1998) calibrated with polystyrene standards. Under the present conditions practically no polymer formation was detected during first $20 \mathrm{~s}$ of irradiation (data not shown), with 
gelation (assessed visually) occurring at around $250 \mathrm{~s}$. Typical electron microscopy images of the materials synthesized for different periods are presented in Figure 1. Maximum yield of polymer with $M_{w} 3-100 \mathrm{kDa}$ was achieved just prior to gelation at $170 \mathrm{~s}$ irradiation. The yield of the required fraction was relatively low, approximately $3 \%(\mathrm{w} / \mathrm{w})$ of the initial mass of monomers. Higher yields were obtained with dilution of the polymerization mixture prior to gelation as reported elswhere (Wulff et al., 2006) (up to $99 \%$ ), although the method relies on thermal polymerisation, and increased temperature during synthesis can have adverse effects on the molecular recognition ability of polymers as demonstrated previously (Piletsky et al., 2002). For that reason UV initiation was used to ensure the formation of high affinity binding sites. Also, the use of high monomer concentration (minimum amount of solvent that enabled template dilution) ensures the formation of stable monomer-template complexes which in turn should result in high affinity imprints, as opposed to precipitation methods which require high dilution (Haupt, 2003). Subsequent fractionation of the nanoparticles (both imprinted and blank particles) by size was performed by SEC, and four fractions collected, with average apparent molecular weights $\left(M_{w}\right)$ of $10-13 \mathrm{kDa}, 60$ $70 \mathrm{kDa}, 90-100 \mathrm{kDa}$ and $120>\mathrm{kDa}$.

\subsection{Presence of residual template.}

No amino residues were detected by the sensitive o-phthaldialdehyde (OPA) method (Paramas et al., 2006) in the purified materials, demonstrating complete removal of the template, which could be derivatised and detected via fluorescent emission at $440 \mathrm{~nm}$. In the present system, template could be detected down to $9 \times 10^{-8} \mathrm{M}$. Template leaching is a common problem associated with imprinted polymers synthesized via bulk polymerization, and it is a considerable obstacle when attempting to use MIPs in systems with very low limits of detection (Olsen et al., 1999; Chassaing et al., 2004).

\subsection{Recognition properties of nanoparticles}


The affinity of polymer fractions was analysed by affinity chromatography on HPLC mode. The type of affinity material used (porous ethylene glycol dimethacrylate-co-DMET) has random polymer branches, some of these will be terminated by a DMET molecule, acting as spacer arms between the polymer network and the affinity ligand. The highest retention time was found for particles with apparent $M_{w}$ of 90-100 kDa (15.41 $\left.\pm 0.43 \mathrm{~min}\right)$, particles with 60-70 and 10-13 kDa had retention times $10.85 \pm 0.07$ and $9.78 \pm 0.12$ min respectively. Blank particles with apparent $M_{w}$ of 90-100 kDa were retained for $7.01 \pm 0.15 \mathrm{~min}$. The same tests were carried out using a nonaffinity phase prepared in the absence of the template analogue, where blank and imprinted nanoparticles had the same retention time $(3.93 \pm 0.04 \mathrm{~min})$ showing no variation in non-specific binding. This also confirms that both imprinted and blank particles have the same hydrodynamic radius, due to the SEC selection step, so the difference in retention times on the affinity column can be assigned to specific interactions with the immobilised template. Tests were performed in triplicate. As the fraction with 90-100 kDa demonstrated higher affinity it was used in subsequent tests, after purification by affinity chromatography (see Supplementary Material). Possibly smaller particles do not have sufficient bulk to accommodate a stable three-dimensional structure of the imprints. This also seems to suggest that the high afinity binding sites are located on the periphery, or on the exterior polymer layers, as smaller particles have retention times which are closer to that of blank particles. Larger nanoparticle fractions $\left(M_{w}>120 \mathrm{kDa}\right)$ had lower retention times than the 90-100 kDa fraction. Possibly these are too bulky to interact properly with the affinity phase, and so cannot be properly selected via affinity chromatography. For this reason larger fractions were not used on subsequent experiments. Figure 2 demonstrates the efficiency of the affinity chromatography in selecting a population of particles with high affinity binding sites. There is a large fraction of particles with low affinity in the mixture after the SEC step as can be seen on Figure 2 on left side. When a high affinity fraction collected from the affinity column is injected again it is clear that the low affinity fractions are not present. For comparison, a chromatogram of blank nanoparticles injected on the affinity column after affinity separation is also displayed. The 
peaks present at the beginning of the chromatograms (approx. from 1.5 to $3 \mathrm{~min}$ ) can be assigned to the presence of nanoparticle clusters due to mechanical entanglement of their three-dimensional network as discussed elsewhere ( $\mathrm{Li}$ et al., 2004). These clusters tend to form in solution and as a consequence, entangled particles might not have their binding sites accessible and so will not be retained.

Further tests were performed with nanoparticles (size range 90-100 kDa) immobilised onto poly(trimethylolpropane trimethacrylate) poly(TRIM) particles by UV irradiation (see Supplementary Material). This approach exploits the living nature of the iniferter-prepared nanoparticles, which allows easy immobilization of the polymer to surfaces containing vinyl groups. Immobilization phase was synthesized by polymerizing cross-linker for the minimum amount of time necessary to obtain a rigid material, but still with a large number of unreacted double bonds. Approximately $36 \%$ of initial double bonds (when compared with liquid TRIM) are still present in the poly(TRIM).

As expected, after 5 minutes of UV irradiation of a solution of nanoparticles mixed with the immobilization phase only $32.9 \%$ of the initial amount of nanoparticles remained in solution, decreasing to $11.2 \%$ after 8 minutes of UV exposure, further irradiation did not considerably decrease the amount of nanoparticles in solution. In total, $0.35 \mathrm{mg}$ imprinted nanoparticles were immobilised per gram of TRIM polymer. The same procedure was repeated with non-imprinted material with similar results (with $11.0 \%$ remaining in solution).

The composite material with immobilised nanoparticles offers the possibility of measuring their binding properties in solution using the double reciprocal method (Chianella et al., 2003). This method was selected as the solvent used in the rebinding experiments was not entirely compatible with techniques such as dialysis, and unspecific adsorption of the template to membranes could also influence the results. Rebinding experiments were only carried out for the high affinity fraction (ca. $56 \%$ of the total fraction of particles with $90-100 \mathrm{kDa}$ ) as the remaining fractions had retention times similar to that of the blank material. Binding experiments were carried out for imprinted and 
non-imprinted nanoparticles. For comparison purposes the same tests were carried out for bulk imprinted polymer prepared with the same composition used to synthesise the imprinted nanoparticles. Template concentration ranged from $8 \mathrm{nM}$ to $1.5 \mathrm{mM}$. According to the double reciprocal plots the dissociation constant $\left(K_{d}\right)$ for imprinted material and the concentration of binding sites in the immobilised fraction $\left(B_{\max }\right)$ were $6.6 \times 10^{-8} \mathrm{M}$ and $8.6 \times 10^{-7} \mathrm{~mol} / \mathrm{g}$ respectively. For the non-imprinted polymer, $K_{d}$ and $B_{\max }$ were $7.9 \times 10^{-6} \mathrm{M}$ and $1.0 \times 10^{-5} \mathrm{~mol} / \mathrm{g}$ respectively. For the imprinted polymer prepared by bulk polymerization $K_{d}$ and $B_{\max }$ were $14 \times 10^{-6} \mathrm{M}$ and 178 $\times 10^{-6} \mathrm{~mol} / \mathrm{g}$. The apparent density of binding sites corresponds to approximately 0.6 binding sites/immobilized imprinted particle, although some binding sites might not be accessible due to the immobilisation. The affinity of the immobilised imprinted nanoparticles is in line with that reported for monoclonal antibodies for atrazine: $K_{d}$ of $3.87 \times 10^{-7} \mathrm{M}\left(\right.$ Grant et al., 1999) and $9.20 \times 10^{-9} \mathrm{M}$. (Kramer, 2002).

For the specificity tests the immobilised nanoparticles were incubated with a solution of either template or structural analogues (atrazine, metribuzin and simazine) in acetonitrile. The amount of each compound adsorbed by the polymer is depicted in Figure 3. Whilst the blank nanoparticles show some preferential binding towards acetoguanamine, this is negligible when compared with the imprinted nanoparticles which have clearly higher affinity and specificity.

\section{Conclusions}

In conclusion, the products synthesized using this approach are a viable and robust alternative to natural receptors, with comparable affinity and specificity, but with much higher stability and ease of preparation. The findings reported here might significantly increase the scope and chances for practical applications of MIP technology.

Acknowledgement. SP would like to acknowledge receiving Royal Society-Wolfson Research Merit Award. EP would like to acknowledge Leverhulme Trust for financial support. 


\section{References}

Chassaing, C., Stokes, J., Venn, R. F., Lanza, F., Sellergren, B., Holmberg, A., Berggren, B., 2004. Journal of Chromatography B 804 (1) 71-81.

Chianella, I., Piletsky, S. A., Tothill, I. E., Chen, B., Turner, A. P. F., 2003. Biosensors \& Bioelectronics 18 (2-3), 119-127.

Grant, S. D., Porter, A. J., Harris, W. J., 1999. Journal of Agricultural and Food Chemistry 47 (1), $340-345$.

Haupt, K., 2003. Analytical Chemistry 75 (17), 376A-383A.

Holthoff, E. L. and Bright, F. V., 2007. Analytica Chimica Acta 594 (2), 147-161.

Kramer, K., 2002. Environmental Science \& Technology 36 (22), 4892-4898.

Li, F. X., Lu, Z. F., Qian, H. T., Rui, J. M., Chen, S. N., Jiang, P., An, Y. L., Mi, H. F., 2004. Macromolecules 37 (3), 764-768.

Mosbach, K. and Ramstrom, O., 1996. Bio-Technology 14 (2), 163-170.

Muldoon, M. T. and Stanker, L. H., 1997. Analytical Chemistry 69 (5), 803-808.

Olsen, J., Martin, P., Wilson, I., Jones, G. R., 1999. The Analyst 124, 467-471.

Paramas, A. M. G., Barez, J. A. G., Marcos, C. C., Garcia-Villanova, R. J., Sanchez, J. S., 2006. Food Chemistry 95 (1), 148-156.

Peppas, N. A. and Ward, J. H., 2004. Advanced Drug Delivery Reviews 56 (11), 1587-1597. 
Piletsky, S. A., Piletska, E. V., Karim, K., Freebairn, K. W., Legge, C. H., Turner, A. P. F., 2002. Macromolecules 35 (19), 7499-7504.

Sergeyeva, T. A., Piletsky, S. A., Brovko, A. A., Slinchenko, E. A., Sergeeva, L. M., El'skaya, A. V., 1999. Analytica Chimica Acta 392 (2-3), 105-111.

Sun, S. S., Zhang, C., Yang, Z. X., Dalton, L. R., Garner, S. M., Chen, A., Steier, W. H., 1998. Polymer 39 (20), 4977-4981.

Wulff, G., Chong, B. O., Kolb, U., 2006. Angewandte Chemie-International Edition 45 (18), 29552958.

Wulff, G., Grobeeinsler, R., Vesper, W., Sarhan, A., 1977. Makromolekulare ChemieMacromolecular Chemistry and Physics 178 (10), 2817-2825.

Zimmerman, S. C., Zharov, I., Wendland, M. S., Rakow, N. A., Suslick, K. S., 2003. Journal of the American Chemical Society 125 (44), 13504-13518.

\section{Figure Captions}




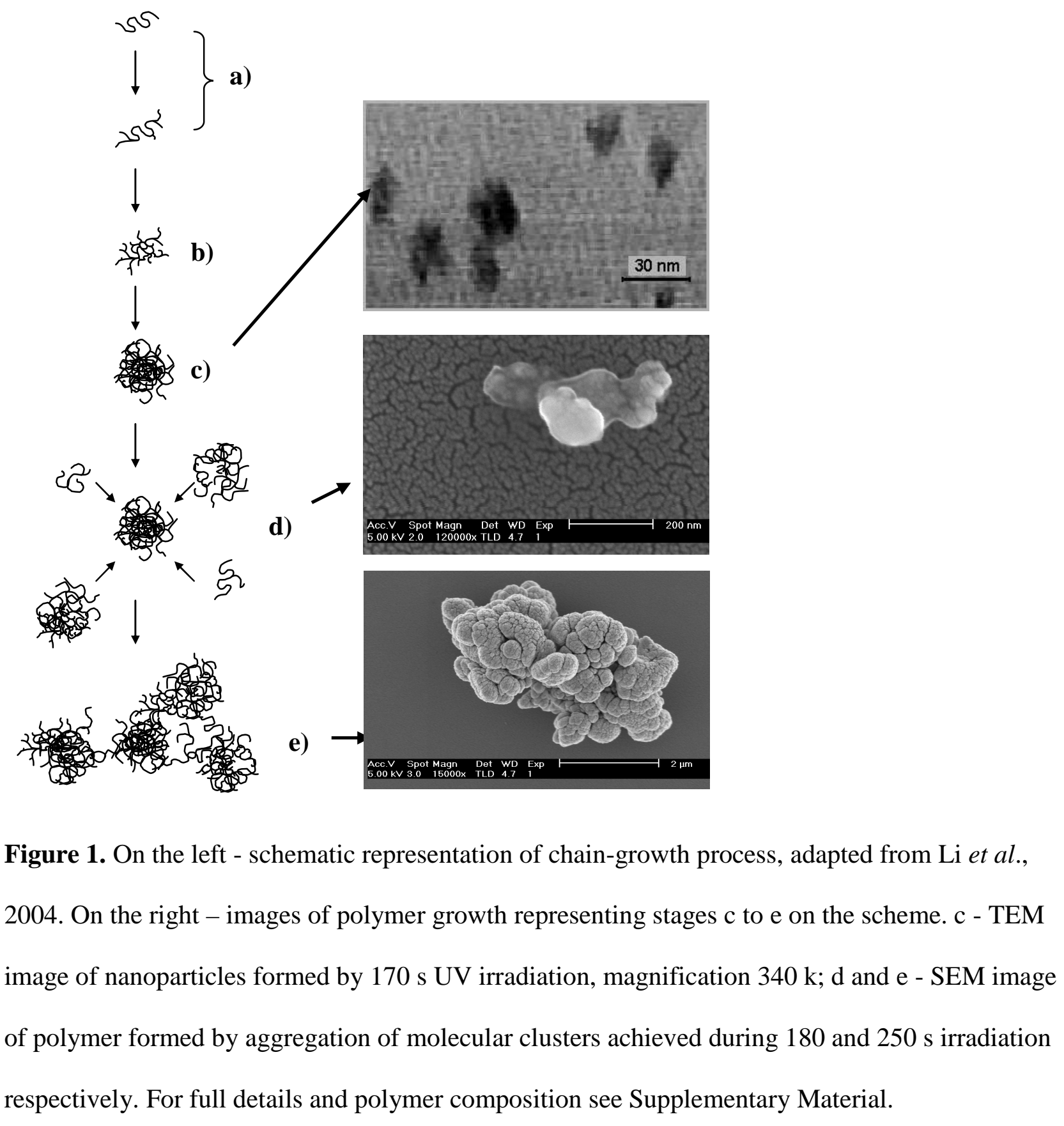



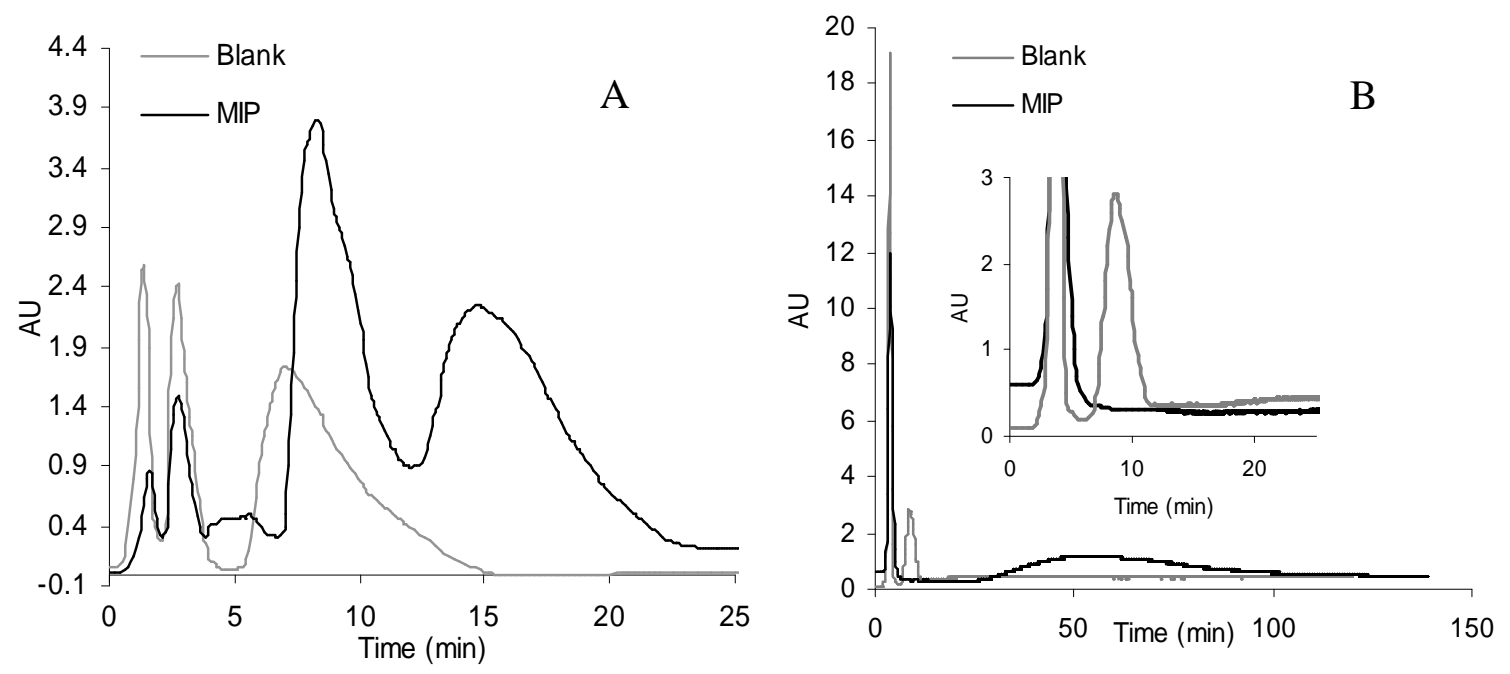

Figure 2. Chromatograms for the blank and imprinted nanoparticles (fraction 90-100 kDa) on the affinity column (A). The chromatograms for the blank and high affinity imprinted nanoparticles (fraction $90-100 \mathrm{kDa}$ ) after affinity purification and injected on the affinity column are depicted in Figure B, the first 25 minutes of these chromatograms are magnified and presented as an inset. 


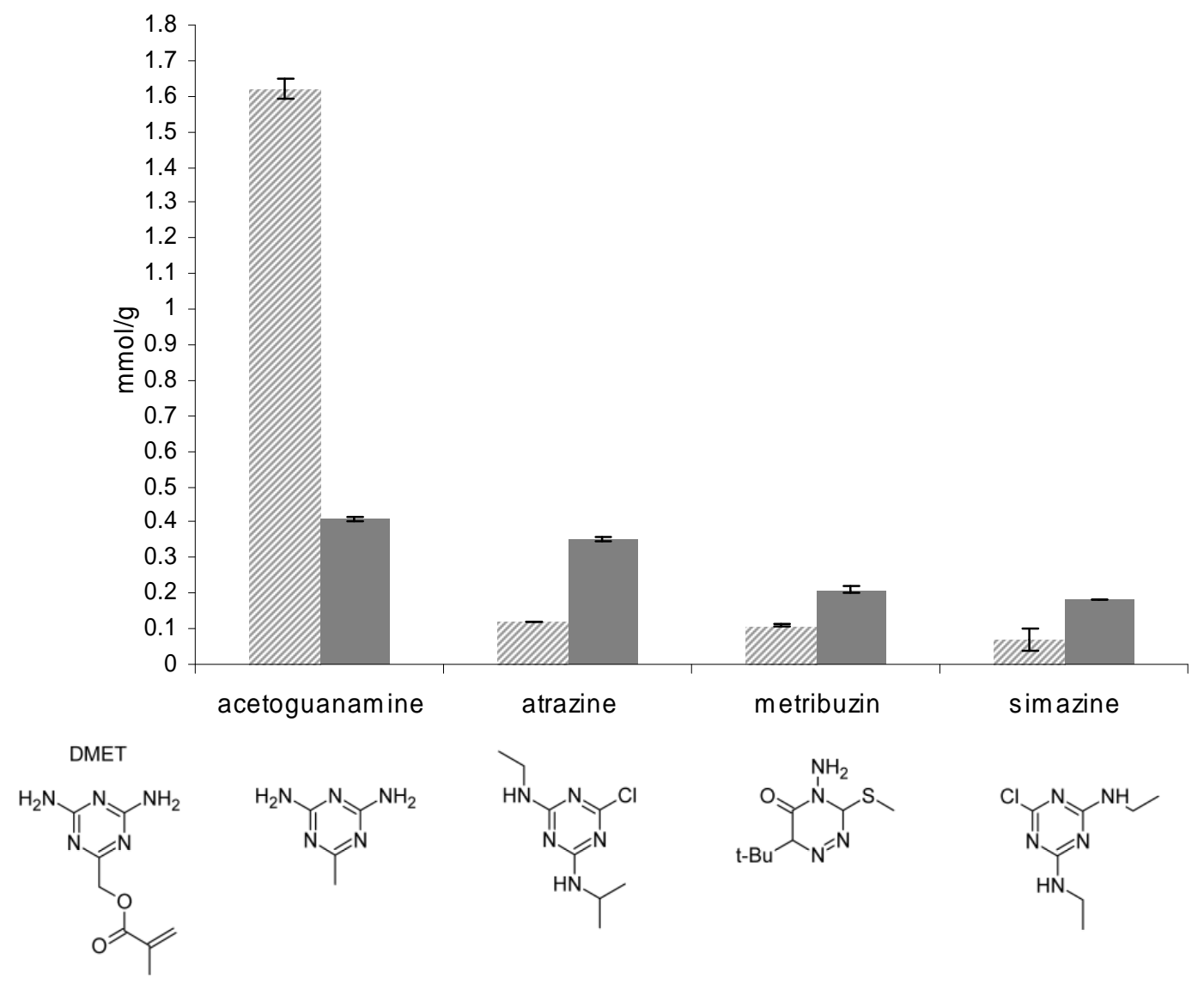

Figure 3. Binding of the template (acetoguanamine) and structural analogues - atrazine, metribuzin and simazine to MIP (light gray bars) and blank (dark gray bars) nanoparticles immobilized on solid support. The structure of the polymerisable template analogue, (DMET) is depicted on the left. Error bars represent the standard deviation $(n=3)$. For these tests, solid phase with the immobilised nanoparticles $(70 \mathrm{mg})$ was placed in $1.5 \mathrm{ml}$ sealed vials with $1 \mathrm{ml}$ solution $(40 \mathrm{ng} / \mathrm{ml})$ of the template (acetoguanamine) or one of its analogues - atrazine, metribuzine or simazine in acetonitrile. Vials were incubated for 12 hours, and the concentration of either chemical that remained in solution was measured by HPLC-MS. See Supplementary Material for full details. 